\title{
Noninvasive ventilation as a weaning strategy for mechanical ventilation in adults with respiratory failure: a Cochrane systematic review
}

\author{
Karen E.A. Burns MD MSc, Maureen O. Meade MD MSc, Azra Premji MSc RRT, Neill K.J. Adhikari MDCM MSc
}

Competing interests: None declared.

This article has been peer reviewed.

This article is based on a Cochrane review published in the Cochrane Database of Systematic Reviews 2013; issue 12, DOI: 10.1002 /14651858.CD004127.pub3

Correspondence to:

Karen Burns,

burnsk@smh.ca

CMAJ 2014. DOI:10.1503 /cmaj.130974

\section{ABSTRACT}

Background: Noninvasive ventilation has been studied as a means of reducing complications among patients being weaned from invasive mechanical ventilation. We sought to summarize evidence comparing noninvasive and invasive weaning and their effects on mortality.

Methods: We identified relevant randomized and quasirandomized trials through searches of databases, conference proceedings and grey literature. We included trials comparing extubation and immediate application of noninvasive ventilation with continued invasive weaning in adults on mechanical ventilation. Two reviewers each independently screened citations, assessed trial quality and abstracted data. Our primary outcome was mortality.

Results: We identified 16 trials involving 994 participants, most of whom had chronic obstructive pulmonary disease (COPD). Compared with invasive weaning, noninvasive weaning significantly reduced mortality (risk ratio $[R R] 0.53,95 \%$ confidence interval $[\mathrm{Cl}]$
0.36 to 0.80 ), weaning failures (RR $0.63,95 \%$ $\mathrm{Cl} 0.42$ to 0.96$)$, ventilator-associated pneumonia (RR $0.25,95 \% \mathrm{Cl} 0.15$ to 0.43 ), length of stay in the intensive care unit (mean difference [MD] $-5.59 \mathrm{~d}, 95 \% \mathrm{Cl}-7.90$ to -3.28 ) and in hospital (MD $-6.04 \mathrm{~d}, 95 \% \mathrm{Cl}-9.22$ to -2.87 ), and total duration of mechanical ventilation (MD $-5.64 \mathrm{~d}, 95 \% \mathrm{Cl}-9.50$ to -1.77$)$. Noninvasive weaning had no significant effect on the duration of ventilation related to weaning, but significantly reduced rates of tracheostomy (RR $0.19,95 \% \mathrm{Cl} 0.08$ to 0.47 ) and reintubation ( $\mathrm{RR} 0.65,95 \% \mathrm{Cl} 0.44$ to 0.97 ). Mortality benefits were significantly greater in trials enrolling patients with COPD than in trials enrolling mixed patient populations (RR 0.36 [95\% Cl 0.24 to 0.56] v. RR 0.81 [95\% Cl 0.47 to 1.40$]$ ).

Interpretation: Noninvasive weaning reduces rates of death and pneumonia without increasing the risk of weaning failure or reintubation. In subgroup analyses, mortality benefits were significantly greater in patients with COPD.
$\mathrm{P}$ atients with acute respiratory failure often require endotracheal intubation and mechanical ventilation to sustain life. Although it is effective, invasive ventilation is associated with complications including respiratory muscle weakness, upper airway pathology, ventilator-associated pneumonia ${ }^{1}$ and sinusitis. ${ }^{2}$ Ventilator-associated pneumonia has been associated with increased morbidity and a trend toward increased mortality. ${ }^{3}$ Consequently, minimizing the duration of invasive mechanical support without increasing the risk of adverse events is an important goal for critical care clinicians. ${ }^{4}$

Noninvasive ventilation may provide a means of reducing the duration of invasive mechanical support for patients with acute respiratory failure. Unlike invasive ventilation, noninvasive ventilation is delivered with an oronasal, nasal or total face mask, or a helmet, connected to a ventilator, and does not require an artificial airway. One can then administer oxygen, augment inhaled volume and apply extrinsic positive end-expiratory pressure to counteract intrinsic positive end-expiratory pressure, similar to invasive ventilation. ${ }^{5}$ Noninvasive ventilation has been shown to augment tidal volume, reduce breathing frequency, rest the muscles of respiration and improve gas exchange. ${ }^{6}$ Randomized controlled trials (RCTs) and metaanalyses have shown that noninvasive ventilation decreases mortality and intubation rates compared with standard medical treatment alone in the treatment of acute exacerbations of chronic obstructive pulmonary disease (COPD). ${ }^{7.8}$

Many patients undergo intubation when noninvasive ventilation has failed or is contraindicated. To mitigate complications associated with protracted invasive ventilation, researchers have investigated the role of noninvasive ventilation in weaning; that is, replacing invasive support with 
noninvasive support in patients who are ready to be weaned but not yet ready for mechanical ventilation to be removed. Because no artificial airway is used with noninvasive ventilation and the cough reflex is preserved, the risk for ventilatorassociated pneumonia is reduced. ${ }^{9,10}$ Additionally, noninvasive weaning may reduce the requirement for sedation, ${ }^{11}$ decrease psychological distress $^{12}$ and permit speech and oral intake. ${ }^{13}$ However, with noninvasive weaning, clinicians must anticipate drying of secretions, accept that only partial ventilatory support can be provided and forfeit a protected airway. Since its initial description as a weaning modality, ${ }^{14}$ RCTs and meta-analyses ${ }^{15,16}$ have compared noninvasive ventilation with alternative weaning strategies. A recent guideline suggested that noninvasive ventilation could be used to facilitate early liberation from mechanical ventilation in patients who have COPD at centres with expertise in its use. ${ }^{17}$ The purpose of this review was to critically appraise, summarize and update a systematic review and meta-analysis of the effect of noninvasive weaning compared with invasive weaning on important outcomes in light of new evidence.

\section{Methods}

\section{Data sources and search criteria}

We updated a previously conducted search of MEDLINE (January 1966 to May 2013) and Embase (January 1980 to May 2013) via OvidSP, the Cochrane Central Register of Controlled Trials (CENTRAL) (The Cochrane Library 2012, Issue 5, 2013) without language restrictions (Appendix 1, available at www.cmaj.ca/lookup/suppl/doi:10 .1503/cmaj.130974/-/DC1). Two reviewers (KB, NA) independently screened citation titles and abstracts. Two reviewers (KB, MM) updated manual searches of abstracts from conference proceedings published in the American Journal of Respiratory and Critical Care Medicine, Intensive Care Medicine, Critical Care Medicine and Chest from April 2009 to May 2013. We reviewed the reference lists of retrieved articles to identify potentially relevant trials, contacted authors to obtain additional information regarding study methods where needed and searched for ongoing trials at Controlled-trials.com and ClinicalTrials.gov. Ethics approval was not required for this systematic review.

\section{Study selection}

We included randomized and quasirandomized trials that enrolled adults with respiratory failure who required invasive mechanical ventilation for at least 24 hours, compared extubation with immediate application of noninvasive ventilation with con- tinued invasive weaning and reported at least one of the following outcomes: mortality (primary outcome), ventilator-associated pneumonia, weaning failure (using authors' definitions), length of stay in the intensive care unit (ICU) or hospital, total duration of ventilation, duration of ventilation related to weaning, duration of invasive ventilation, adverse events or quality of life. We excluded trials that compared noninvasive weaning with invasive weaning in the immediate postoperative setting, compared noninvasive ventilation with unassisted oxygen supplementation or investigated noninvasive ventilation after unplanned extubation.

\section{Data extraction and quality assessment}

Two authors (KB, NA), unblinded to the source of the reports, abstracted data regarding study methods (randomization, allocation concealment, completeness of follow-up, selective outcomes reporting) using a standardized form. Disagreements regarding study selection and data abstraction were resolved by consensus and arbitration with a third author (MM).

\section{Data synthesis and statistical analysis}

We pooled data across studies using random effects models. We derived summary estimates of risk ratio (RR) and mean difference (MD) with $95 \%$ confidence intervals (CIs) for binary and continuous outcomes, respectively, using Review Manager 5.1.6. ${ }^{18}$ If an outcome was reported at 2 different times, we included the more protracted measure in pooled analyses.

We evaluated the effect of statistical heterogeneity among pooled studies for each outcome using the Cochran $Q$ statistic (threshold $p<0.10)^{19,20}$ and the $I^{2}$ test $^{21,22}$ with threshold values of $0 \%-40 \%$ (representing heterogeneity that might not be important), and 30\%-60\%, 50\%-90\%, and $\geq 75 \%$ (representing moderate, substantial or considerable heterogeneity, respectively). ${ }^{22}$ If a heterogeneity value overlapped 2 categories, we assigned it the higher rating. In sensitivity analyses, we assessed the effect of excluding quasirandomized trials on estimates of mortality and ventilator-associated pneumonia. We planned subgroup analyses to compare the effects of noninvasive weaning on mortality and weaning failure in studies including only patients with COPD with the effects seen in studies involving patients without COPD or mixed populations. In addition, we compared the effects seen in studies in which at least $50 \%$ of the participants had COPD with the effects seen in studies in which less than $50 \%$ of participants had COPD. We assessed for differences between subgroup summary estimates using the $\chi^{2}$ test. $^{23}$ We used the GRADE (Grades of Recommendation, Assessment, Development and Evaluation) principles ${ }^{24}$ to assess the 
quality of evidence associated with specific outcomes (mortality, weaning failure, ventilatorassociated pneumonia, duration of ventilation related to weaning and reintubation).

\section{Results}

\section{Trial identification}

We identified 1506 records in our updated search. Of the 961 unique records we found, we assessed 15 new articles for eligibility (Figure 1). ${ }^{16}$ Although we identified 6 additional trials from our updated search, 1 author confirmed that his trial had been aborted and never published, and 1 trial had not been consistently randomized (see Appendix 2 for a list of the excluded studies, available at www.cmaj.ca/lookup /suppl/doi:10.1503/cmaj.130974/-/DC1). Consequently, we included 4 newly identified trials in our analysis, ${ }^{25-28}$ in addition to the 12 trials included in our previous review. ${ }^{16}$

Of the 16 included trials, 2 were published only in abstract form, ${ }^{29,30} 4$ were published in Chinese, ${ }^{31-34}$ 1 was a dissertation subsequently published in full,,$^{35}$ and one was a pilot $\mathrm{RCT} .{ }^{28}$ We excluded
20 studies (11 identified through our updated search [Figure 1] and 9 excluded previously; Appendix 2), including 9 newly identified publications, 1 abstract and the aborted trial(Figure 1).

Of the 16 included RCTs (involving a total of 994 patients), 9 trials exclusively involved patients with COPD, ${ }^{26,30-37}$ and 7 trials ${ }^{25,27,28,29,38-40}$ included mixed or non-COPD populations (Table 1). In the trials involving mixed or non-COPD patient populations, COPD was diagnosed in about $75 \%$ of patients in 3 trials, ${ }^{25,38,39}$ in about $30 \%$ of patients in 2 trials $^{29,40}$ and in more than $20 \%$ of patients in 1 trial; ${ }^{27}$ COPD was an exclusion criterion in 1 trial. ${ }^{28}$ Patients were considered difficult to wean in 2 trials $^{25,38}$ and had persistent weaning failures in 1 trial. ${ }^{39}$ Four trials ${ }^{32-34,37}$ included patients with COPD whose respiratory failure was due to pulmonary infection. The 2 reviewers achieved complete agreement on study selection.

\section{Quality assessment}

Overall, the quality of the included trials was moderate to good (Tables 2 and 3). In most of the trials, allocation to the treatment group was by random assignment, with 1 quasirandomized trial

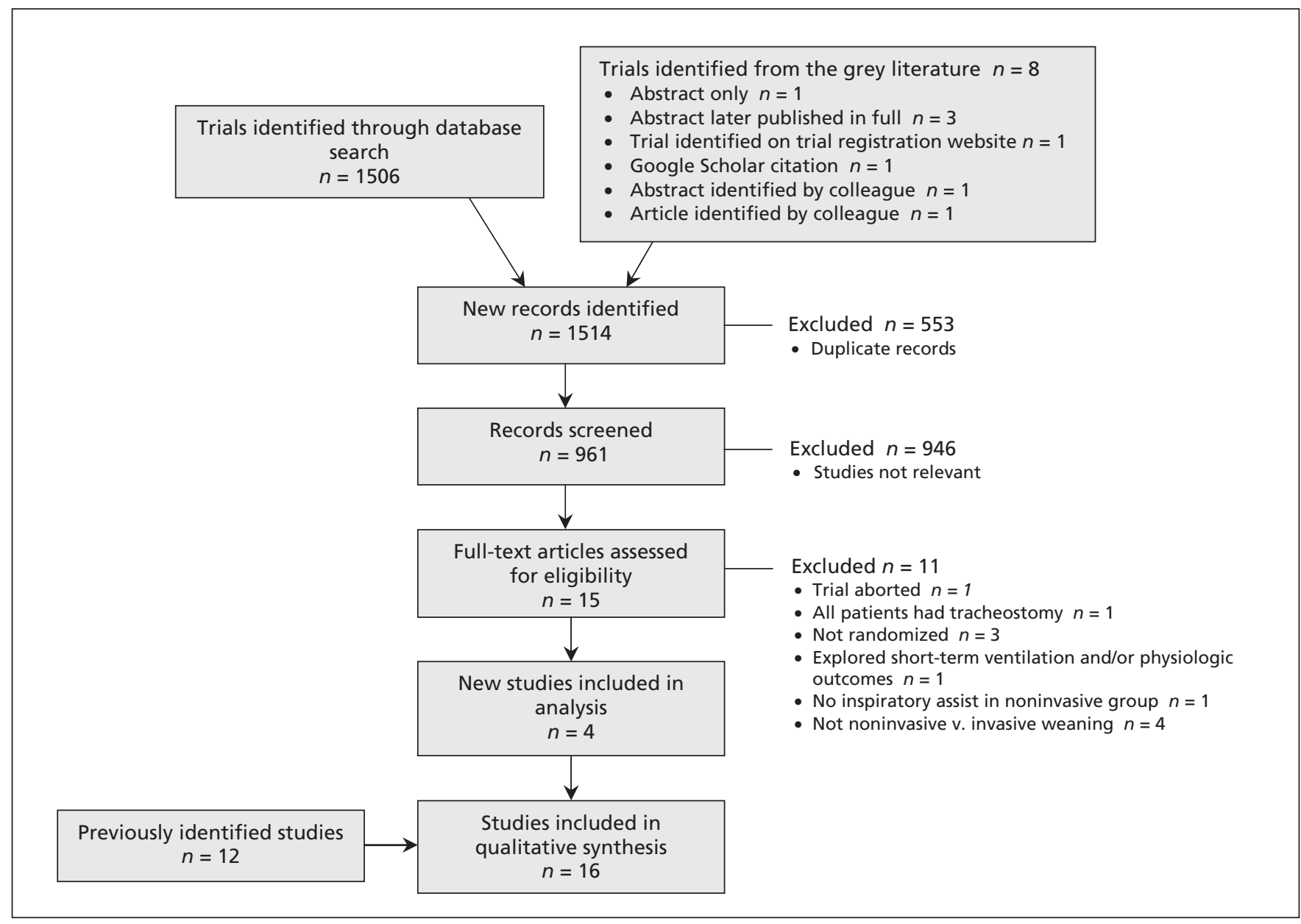

Figure 1: Selection of included studies. This review represents an update of a previously conducted systematic review and meta-analysis. ${ }^{16}$ 
Table 1: Populations and interventions in studies of noninvasive ventilation in adults with critical illness

\begin{tabular}{|c|c|c|c|c|c|}
\hline \multirow[b]{2}{*}{ Study } & \multirow{2}{*}{$\begin{array}{l}\text { No. of } \\
\text { patients }\end{array}$} & \multicolumn{2}{|l|}{ Inclusion criteria } & \multirow[b]{2}{*}{ Experimental strategy } & \multirow[b]{2}{*}{ Control strategy } \\
\hline & & Patient characteristics & Weaning eligibility & & \\
\hline Girault et al. $2011^{25}$ & 138 & $\begin{array}{l}\text { Chronic hypercapnic respiratory failure; } \\
\text { invasive mechanical ventilation for at } \\
\text { least } 48 \mathrm{~h}\end{array}$ & 2 h SBT failure & $\begin{array}{l}\text { Noninvasive pressure } \\
\text { support } \pm \text { PEEP or bilevel } \\
\text { NIV with face mask } \\
\text { (initial choice) }\end{array}$ & $\begin{array}{l}\text { Invasive pressure } \\
\text { support with once } \\
\text { daily SBT with T- } \\
\text { piece or pressure } \\
\text { support } \pm \text { PEEP }\end{array}$ \\
\hline $\begin{array}{l}\text { Rabie Agmy et al. } \\
2012^{26}\end{array}$ & 264 & Acute-on-chronic exacerbation of COPD & 2 h SBT failure & NIV (pressure, ST mode) & $\begin{array}{l}\text { Invasive pressure } \\
\text { support }\end{array}$ \\
\hline $\begin{array}{l}\text { Tawfeek et al. } \\
2012^{27}\end{array}$ & 42 & Invasive mechanical ventilation $>48 \mathrm{~h}$ & 2 h SBT failure & $\begin{array}{l}\text { Noninvasive PAV } \\
\text { delivered by face mask }\end{array}$ & SIMV \\
\hline $\begin{array}{l}\text { Vaschetto et al. } \\
2012^{28}\end{array}$ & 20 & $\begin{array}{l}\text { Hypoxemic respiratory failure; invasive } \\
\text { mechanical ventilation for at least } 48 \mathrm{~h}\end{array}$ & $\begin{array}{l}\text { Pressure support } \\
\text { with PEEP + } \\
\text { inspiratory support, } \\
\leq 25 \mathrm{~cm} \mathrm{H}_{2} \mathrm{O} \text { and } \\
\mathrm{PEEP} 8-13 \mathrm{~cm} \mathrm{H} \mathrm{H}_{2} \mathrm{O} \\
\mathrm{PaO}_{2}: \mathrm{FiO}_{2} 200- \\
300 \mathrm{~mm} \mathrm{Hg}_{-} \text {with } \mathrm{FiO}_{2} \\
\leq 0.6\end{array}$ & Helmet NIV & $\begin{array}{l}\text { Invasive pressure } \\
\text { support with SBT } \\
\text { when } \mathrm{PaO}_{2}: \mathrm{FiO}_{2} \\
>250 \mathrm{~mm} \mathrm{Hg}\end{array}$ \\
\hline Hill et al. $2000^{29}$ & 21 & Acute respiratory failure & 30 min SBT failure & $\begin{array}{l}\text { NIV using VPAP in ST-A } \\
\text { mode }\end{array}$ & $\begin{array}{l}\text { Invasive pressure } \\
\text { support }\end{array}$ \\
\hline $\begin{array}{l}\text { Rabie Agmy et al. } \\
2004^{30}\end{array}$ & 37 & Exacerbation of COPD & 2 h SBT failure & $\begin{array}{l}\text { NIV (proportional assist } \\
\text { in timed mode) delivered } \\
\text { by face or nasal mask }\end{array}$ & $\begin{array}{l}\text { Invasive pressure } \\
\text { support }\end{array}$ \\
\hline Chen et al. $2001^{31}$ & 24 & $\begin{array}{l}\text { Exacerbation of COPD; mechanical } \\
\text { ventilation for at least } 48-60 \mathrm{~h} \text {; } \\
\mathrm{O}_{2} \text { saturation } \geq 88 \% \text { on } \mathrm{FiO}_{2} 40 \%\end{array}$ & $\begin{array}{l}\text { Day } 3+\text { weaning } \\
\text { criteria }\end{array}$ & $\begin{array}{l}\text { Bilevel NIV (pressure } \\
\text { mode) }\end{array}$ & $\begin{array}{l}\text { Invasive pressure } \\
\text { support }\end{array}$ \\
\hline Wang et al. $2004^{32 *}$ & 28 & COPD; bronchopulmonary infection & PIC window & $\begin{array}{l}\text { NIV (pressure mode) } \\
\text { delivered by mask } \\
\text { (unspecified) }\end{array}$ & $\begin{array}{l}\text { SIMV + pressure } \\
\text { support }\end{array}$ \\
\hline Zheng et al. $2005^{33 *}$ & 33 & COPD; severe pulmonary infection & PIC window & $\begin{array}{l}\text { Bilevel NIV (pressure } \\
\text { mode) delivered by face } \\
\text { or nasal mask }\end{array}$ & $\begin{array}{l}\text { Invasive pressure } \\
\text { support }\end{array}$ \\
\hline Zou et al. $2006^{34 *}$ & 76 & $\begin{array}{l}\text { COPD with severe respiratory failure; } \\
\text { pulmonary infection }\end{array}$ & PIC window & $\begin{array}{l}\text { Bilevel NIV (pressure, ST } \\
\text { mode) delivered by nasal } \\
\text { or oronasal mask }\end{array}$ & $\begin{array}{l}\text { SIMV + pressure } \\
\text { support }\end{array}$ \\
\hline Prasad et al. $2009^{35}$ & 30 & COPD; hypercapnic respiratory failure & 2 h SBT failure & $\begin{array}{l}\text { Bilevel NIV (pressure } \\
\text { mode) delivered by full } \\
\text { face mask }\end{array}$ & $\begin{array}{l}\text { Invasive pressure } \\
\text { support }\end{array}$ \\
\hline Nava et al. $1998^{36}$ & 50 & $\begin{array}{l}\text { Exacerbation of COPD; mechanical } \\
\text { ventilation for at least } 36-48 \mathrm{~h}\end{array}$ & $\begin{array}{l}\text { Simple weaning } \\
\text { criteria, } 1 \mathrm{~h} \text { SBT } \\
\text { failure }\end{array}$ & $\begin{array}{l}\text { Noninvasive pressure } \\
\text { support on conventional } \\
\text { ventilator delivered with } \\
\text { face mask }\end{array}$ & $\begin{array}{l}\text { Invasive pressure } \\
\text { support }\end{array}$ \\
\hline $\begin{array}{l}\text { Collaborating } \\
\text { Research Group for } \\
\text { Noninvasive } \\
\text { Mechanical } \\
\text { Ventilation } 2005^{37 *}\end{array}$ & 90 & $\begin{array}{l}\text { COPD with severe hypercapnic } \\
\text { respiratory failure; pneumonia or } \\
\text { purulent bronchitis; age } \leq 85 \text { y; capable } \\
\text { of self-care during previous year }\end{array}$ & PIC window & $\begin{array}{l}\text { Bilevel NIV (pressure } \\
\text { mode) }\end{array}$ & $\begin{array}{l}\text { SIMV + pressure } \\
\text { support }\end{array}$ \\
\hline Girault et al. $1999^{38}$ & 33 & $\begin{array}{l}\text { Acute-on-chronic respiratory failure } \\
\text { (COPD, restrictive or mixed } \\
\text { populations); mechanical ventilation } \\
\text { for at least } 48 \mathrm{~h}\end{array}$ & $\begin{array}{l}\text { Simple weaning } \\
\text { criteria, } 2 \text { h SBT } \\
\text { failure }\end{array}$ & $\begin{array}{l}\text { Flow or pressure mode } \\
\text { with nasal or face mask }\end{array}$ & $\begin{array}{l}\text { Flow or pressure } \\
\text { mode (pressure } \\
\text { support) }\end{array}$ \\
\hline Ferrer et al. $2003^{39}$ & 43 & $\begin{array}{l}\text { Acute respiratory failure and persistent } \\
\text { weaning failure; intubation for at least } \\
72 \mathrm{~h}\end{array}$ & $\begin{array}{l}2 \text { h SBT failure on } \\
3 \text { consecutive days }\end{array}$ & $\begin{array}{l}\text { Bilevel NIV in ST mode } \\
\text { delivered with face or } \\
\text { nasal mask }\end{array}$ & $\begin{array}{l}\text { Assist control or } \\
\text { invasive pressure } \\
\text { support }\end{array}$ \\
\hline Trevisan et al. $2008^{40}$ & 65 & Invasive mechanical ventilation $>48 \mathrm{~h}$ & 30 min SBT failure & $\begin{array}{l}\text { Bilevel NIV (pressure } \\
\text { mode) delivered by } \\
\text { facemask }\end{array}$ & $\begin{array}{l}\text { Invasive } \\
\text { mechanical } \\
\text { ventilation }\end{array}$ \\
\hline \multicolumn{6}{|c|}{ 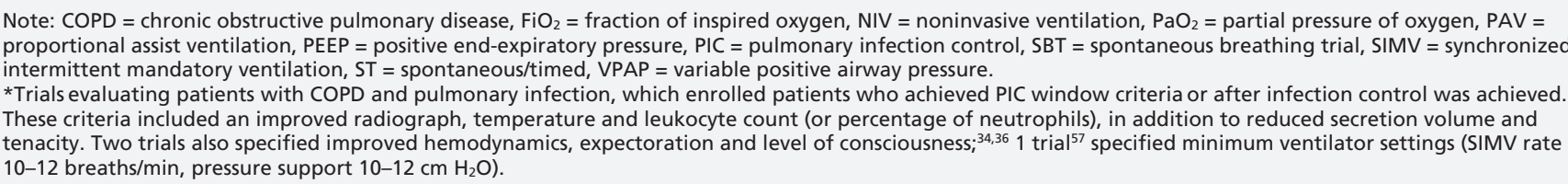 } \\
\hline
\end{tabular}


allocating patients according to order of hospital admission. ${ }^{31}$ We judged allocation concealment to be adequate in 8 trials, ${ }^{25-30,36,39}$ unclear in 7 tri- als $\mathrm{s}^{32-35,37,38,40}$ and inadequate in 1 quasirandomized trial. ${ }^{31}$ In 2 trials, ${ }^{32,34}$ denominators were not provided in binary outcomes to ensure complete

Table 2: Risk of bias in the included trials

\begin{tabular}{|c|c|c|c|c|}
\hline \multirow[b]{2}{*}{ Study } & \multicolumn{2}{|c|}{ Selection bias } & \multirow[b]{2}{*}{$\begin{array}{l}\text { Attrition bias } \\
\text { (incomplete } \\
\text { data) }\end{array}$} & \multirow[b]{2}{*}{$\begin{array}{l}\text { Reporting bias } \\
\text { (selective } \\
\text { reporting) }\end{array}$} \\
\hline & $\begin{array}{c}\text { Random } \\
\text { sequence } \\
\text { generation }\end{array}$ & $\begin{array}{l}\text { Allocation } \\
\text { concealment }\end{array}$ & & \\
\hline Girault et al. $2011^{25}$ & Low & Low & Low & Low \\
\hline Rabie Agmy et al. $2012^{26}$ & Low & Low & Low & Low \\
\hline Tawfeek et al. $2012^{27}$ & Unclear & Low & Low & Low \\
\hline Vaschetto et al. $2012^{28}$ & Low & Low & Low & Low \\
\hline Hill et al. $2000^{29}$ & Unclear & Low & Low & Low \\
\hline Rabie Agmy et al. $2004^{30}$ & Unclear & Low & Low & Low \\
\hline Chen et al. $2001^{31}$ & High & High & Low & Unclear \\
\hline Wang et al. $2004^{32}$ & Unclear & Unclear & Low & Low \\
\hline Zheng et al. $2005^{33}$ & Unclear & Unclear & Unclear & Low \\
\hline Zou et al. $2006^{34}$ & Low & Unclear & Unclear & Low \\
\hline Prasad et al. $2009^{35}$ & Low & Unclear & Low & Low \\
\hline Nava et al. $1998^{36}$ & Unclear & Low & Low & Low \\
\hline $\begin{array}{l}\text { Collaborating Research } \\
\text { Group for Noninvasive } \\
\text { Mechanical Ventilation } \\
2005^{37}\end{array}$ & Unclear & Unclear & Low & Low \\
\hline Girault et al. $1999^{38}$ & Unclear & Unclear & Low & Low \\
\hline Ferrer et al. $2003^{39}$ & Low & Low & Low & High \\
\hline Trevisan et al. $2008^{40}$ & Unclear & Unclear & Low & Low \\
\hline
\end{tabular}

Table 3: Summary estimates of effect of noninvasive ventilation in adults with critical illness

\begin{tabular}{|c|c|c|c|c|}
\hline Outcome & $\begin{array}{l}\text { No. of studies } \\
\text { (no. of patients*) }\end{array}$ & \multicolumn{2}{|c|}{$\begin{array}{l}\text { Summary estimate } \\
(95 \% \mathrm{Cl})\end{array}$} & $\begin{array}{l}\text { Heterogeneity, } \\
I^{2}, \%\end{array}$ \\
\hline Death & $16(994)$ & $0.53 \ddagger$ & $(0.36$ to 0.80$)$ & 37 \\
\hline VAP & $14(953)$ & $0.25 \ddagger$ & (0.15 to 0.43$)$ & 38 \\
\hline Weaning failure & $8(605)$ & $0.63 \ddagger$ & (0.42 to 0.96$)$ & 39 \\
\hline \multicolumn{5}{|l|}{ Length of stay } \\
\hline Intensive care unit & $13(907)$ & $-5.59 \S$ & $(-7.90$ to -3.28$)$ & 77 \\
\hline Hospital & $10(803)$ & $-6.04 \S$ & $(-9.22$ to -2.87$)$ & 78 \\
\hline \multicolumn{5}{|c|}{ Duration of mechanical ventilation } \\
\hline Total & $7(385)$ & $-5.64 \S$ & $(-9.50$ to -1.77$)$ & 86 \\
\hline Related to weaning & $9(645)$ & $-0.25 \S$ & $(-2.06$ to 1.56$)$ & 90 \\
\hline Endotracheal† & $12(717)$ & $-7.44 \S$ & $(-10.34$ to -4.55$)$ & 87 \\
\hline \multicolumn{5}{|l|}{ Adverse events } \\
\hline Reintubation & $10(789)$ & $0.65 \ddagger$ & (0.44 to 0.97$)$ & 41 \\
\hline Tracheostomy & $7(572)$ & $0.19 \ddagger$ & (0.08 to 0.47$)$ & 10 \\
\hline Arrhythmia & $3(201)$ & $0.89 \ddagger$ & (0.34 to 2.34$)$ & 0 \\
\hline \multicolumn{5}{|c|}{$\begin{array}{l}\text { Note: } \mathrm{Cl}=\text { confidence interval, VAP = ventilator-associated pneumonia. } \\
\text { *For weaning failure, reintubation and tracheostomy, the numbers of patients in the denominators differ from sums of numbers in } \\
\text { Table } 1 \text { because one study } 38 \text { reported these outcomes differently (i.e., weaning failure included reintubation or death within } 7 \mathrm{~d} \text {; } \\
\text { reintubation included only reintubation within } 7 \mathrm{~d} \text {; tracheostomy was reported in } 105 \text { surviving patients at discharge). } \\
\text { tInvasive ventilation. } \\
\text { fRisk ratio. } \\
\text { §Mean difference. }\end{array}$} \\
\hline
\end{tabular}


reporting. The possibility of selective outcomes reporting was not excluded in 1 trial $^{31}$ that reported clinically important outcomes, but did not specify primary and secondary outcomes. Another triall ${ }^{39}$ did not report weaning outcomes in the full publication, but did report them in a previously published abstract; the authors affirmed that they had not continued to collect these data (Appendix 3, available at www.cmaj.ca /lookup/suppl/doi:10.1503/cmaj.130974/-/DC1).

\section{Primary outcome}

All of the trials provided mortality data, which was reported at 30, 60 and 90 days, ${ }^{27,35,36,38,39}$ at discharge from the ICU $^{25,28}$ or hospital ${ }^{26,28,30,33,34,37,38,40}$ or at an undefined time. ${ }^{29,31,32}$ There was strong evidence that noninvasive weaning reduced mortality (RR 0.53, 95\% CI 0.36 to $0.80 ; 994$ patients) with moderate heterogeneity $\left(I^{2}=37 \% ; p=0.07\right.$ ) (Figure 2 and Appendix 4, available at www.cmaj.ca /lookup/suppl/doi:10.1503/cmaj.130974/-/DC1).

\section{Secondary outcomes}

Eight trials involving 605 patients, using variable definitions, reported the proportion of patients successfully weaned..$^{25-30,36,38}$ The pooled data showed a significant reduction in the proportion of weaning failures using noninvasive weaning (RR 0.63 , 95\% CI 0.42 to 0.96) with moderate heterogeneity $\left(I^{2}=38 \% ; p=0.1\right)$ (Figure 3 ).

Pooled data from 14 trials (involving 953 patients) $)^{25-27,30-40}$ that reported ventilator-associated pneumonia (for which criteria for the diagnosis were provided in 10 trials $^{27,31-37,39,40}$ ) showed that noninvasive weaning was associated with decreased ventilator-associated pneumonia (RR $0.25,95 \%$ CI 0.15 to 0.43$)$, with moderate heterogeneity $\left(I^{2}=38 \% ; p=0.07\right)$ (Figure 4, Appendix 4).

Noninvasive weaning significantly reduced the length of stay in both the ICU (MD -5.59d, $95 \%$ CI -7.90 to -3.28$)$ and the hospital (MD $-6.04 \mathrm{~d}, 95 \% \mathrm{CI}-9.22$ to -2.87 ), the total

\begin{tabular}{|c|c|c|c|c|c|c|c|c|c|}
\hline \multirow{3}{*}{$\begin{array}{l}\text { Subgroup } \\
\text { and study } \\
\text { COPD }\end{array}$} & \multicolumn{2}{|c|}{ Noninvasive } & \multicolumn{2}{|c|}{ Invasive } & \multirow[b]{2}{*}{$\operatorname{RR}(95 \% \mathrm{CI})$} & \multirow{2}{*}{\multicolumn{3}{|c|}{$\begin{array}{r:l}\text { Favours } & \text { Favours } \\
\text { noninvasive } & \text { invasive }\end{array}$}} & \\
\hline & \multirow{2}{*}{\multicolumn{2}{|c|}{$\begin{array}{l}\text { No. of } \begin{array}{c}\text { No. of } \\
\text { events patients }\end{array} \\
\end{array}$}} & \multirow{2}{*}{\multicolumn{2}{|c|}{$\begin{array}{l}\text { No. of No. of } \\
\text { events patients }\end{array}$}} & & & & & \\
\hline & & & & & (1) & & & & \\
\hline Chen et al. $2001^{31}$ & 0 & 12 & 3 & 12 & $0.14(0.01$ to 2.50$)$ & & & & \\
\hline Nava et al. $1998^{36}$ & 2 & 25 & 7 & 25 & $0.29(0.07$ to 1.24$)$ & & & & \\
\hline Prasad et al. $2009^{35}$ & 5 & 15 & 9 & 15 & $0.56(0.24$ to 1.27$)$ & & & & \\
\hline Rabie Agmy et al. $2004^{30}$ & 1 & 19 & 2 & 18 & $0.47(0.05$ to 4.78$)$ & & & & \\
\hline Rabie Agmy et al. $2012^{26}$ & 7 & 134 & 26 & 130 & $0.26(0.12$ to 0.58$)$ & & $\varpi$ & & \\
\hline Wang et al. $2004^{32}$ & 1 & 14 & 2 & 14 & $0.50(0.05$ to 4.90$)$ & & & & \\
\hline CRGNMV $2005^{37}$ & 1 & 47 & 7 & 43 & $0.13(0.02$ to 1.02$)$ & & & & \\
\hline Zheng et al. $2005^{33}$ & 3 & 17 & 3 & 16 & $0.94(0.22$ to 4.00$)$ & & & & \\
\hline Zou et al. $2006^{34}$ & 3 & 38 & 11 & 38 & $0.27(0.08$ to 0.90$)$ & & & & \\
\hline Subtotal & & 321 & & 311 & $0.36(0.24$ to 0.56$)$ & & & & \\
\hline $\begin{array}{l}\text { Total events } \\
R^{2}=0 \%\end{array}$ & 23 & & 70 & & & & & & \\
\hline \multicolumn{10}{|l|}{ Mixed } \\
\hline Ferrer et al. $2003^{39}$ & 6 & 21 & 13 & 22 & $0.48(0.23$ to 1.03$)$ & & & & \\
\hline Girault et al. $1999^{38}$ & 0 & 17 & 2 & 16 & 0.19 (0.01 to 3.66$)$ & & & & \\
\hline Girault et al. $2011^{25}$ & 16 & 69 & 9 & 69 & 1.78 (0.84 to 3.75$)$ & & & & \\
\hline Hill et al. $2000^{29}$ & 1 & 12 & 1 & 9 & $0.75(0.05$ to 10.44$)$ & & & & \\
\hline Tawfeek et al. $2012^{27}$ & 2 & 21 & 6 & 21 & $0.33(0.08$ to 1.47$)$ & & & & \\
\hline Trevisan et al. $2008^{40}$ & 9 & 28 & 10 & 37 & $1.19(0.56$ to 2.53$)$ & & & & \\
\hline Vaschetto et al. $2012^{28}$ & 2 & 10 & 3 & 10 & $0.67(0.14$ to 3.17$)$ & & & & \\
\hline Subtotal & & 178 & & 184 & $0.81(0.47$ to 1.40$)$ & & & & \\
\hline $\begin{array}{l}\text { Total events } \\
R^{2}=35 \%\end{array}$ & 36 & & 44 & & & & & & \\
\hline Total & & 499 & & 495 & $0.53(0.36$ to 0.80$)$ & & $\vartheta$ & & \\
\hline $\begin{array}{l}\text { Total events } \\
I^{2}=37 \%\end{array}$ & 59 & & 114 & & & & & & \\
\hline \multirow{2}{*}{\multicolumn{6}{|c|}{ Test for subgroup differences $p=0.02, I^{2}=80.5 \%$}} & 0.001 & 0.11 & 110 & 1000 \\
\hline & & & & & & \multicolumn{4}{|c|}{$\operatorname{RR}(95 \% \mathrm{CI})$} \\
\hline
\end{tabular}

Figure 2: Effect of noninvasive weaning on mortality. $\mathrm{Cl}=$ confidence interval, $\mathrm{COPD}=$ chronic obstructive pulmonary disease, CRGNMV = Collaborating Research Group for Noninvasive Mechanical Ventilation, RR = risk ratio. 
duration of mechanical ventilation (MD -5.64 d, $95 \% \mathrm{CI}-9.50$ to -1.77$)$ and the duration of invasive ventilation (MD $-7.44 \mathrm{~d}, 95 \% \mathrm{CI}-10.34$ to $-4.55)$, all with considerable heterogeneity. Noninvasive weaning had no effect on the duration of mechanical ventilation related to weaning (MD $-0.25 \mathrm{~d}, 95 \% \mathrm{CI}-2.06$ to 1.56 ). None of the included studies reported on quality of life (Table 3).

\section{Adverse events}

The pooled result showed no difference in arrhythmias (RR 0.89, 95\% CI 0.34 to 2.34; 3 trials, 201 patients), ${ }^{25,35,38}$ but significantly lower rates of reintubation (RR $0.65,95 \%$ CI 0.44 to 0.97 ; 10 trials, 789 patients) ${ }^{25-29,34,37-40}$ and tracheostomy (RR 0.19, 95\% CI 0.08 to $0.47 ; 7$ trials, 572 patients $)^{25-28,38-40}$ with variable heterogeneity (Table 3 ).

\section{Sensitivity and subgroup analyses}

The exclusion of a quasirandomized trial ${ }^{31}$ maintained significant reductions in mortality (RR $0.60,95 \%$ CI 0.40 to 0.90 ) and the rate of ventilator-associated pneumonia (RR $0.27,95 \%$ CI 0.16 to 0.45 ), favouring noninvasive weaning.
We noted a significant difference in RR between subgroups $(p=0.02)$ evaluating the effect of noninvasive weaning on mortality in COPD (RR $0.36,95 \%$ CI 0.24 to 0.56 ; 9 trials) compared with the effect in a mixed population (RR $0.81,95 \%$ CI 0.47 to $1.40 ; 7$ trials). A subgroup analysis comparing trials in which at least $50 \%$ of the enrolled participants had COPD (RR $0.47,95 \%$ CI 0.29 to $0.76 ; 12$ trials) with trials in which less than $50 \%$ of participants had COPD (RR $0.86,95 \%$ CI 0.47 to $1.58 ; 4$ trials) showed a greater reduction in mortality in the COPD-predominant trials. However, the difference was not significant $(p=0.1)$. The effect of noninvasive weaning on weaning failure did not differ significantly between trials involving patients with COPD or mixed populations.

\section{Interpretation}

We identified 16 trials of moderate to good quality comparing noninvasive and invasive weaning among 994 patients, most of whom had COPD. Compared with invasive weaning, noninvasive weaning significantly decreased mortality, the rates

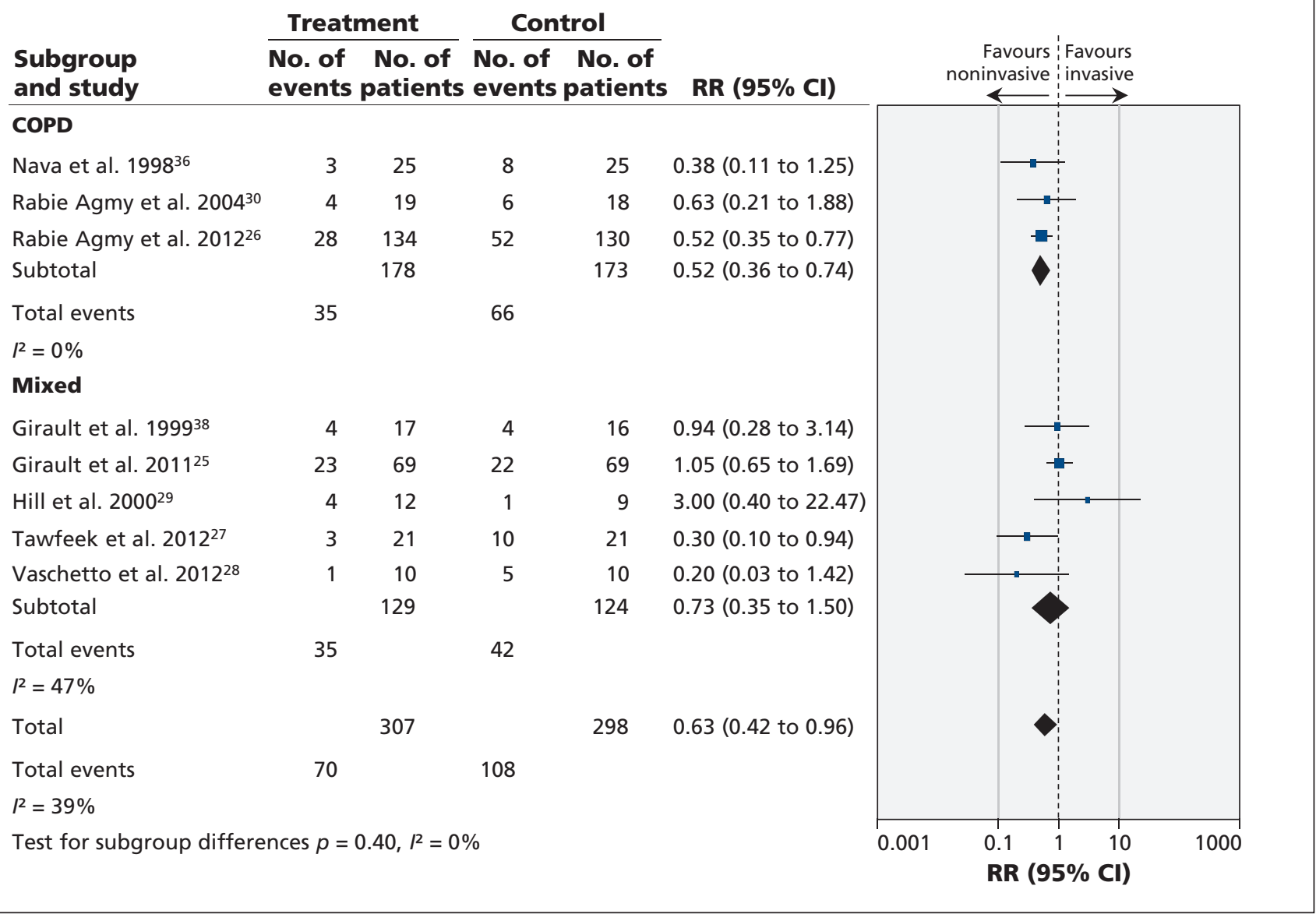

Figure 3: Effect of noninvasive weaning on weaning failures. $\mathrm{Cl}=$ confidence interval, $\mathrm{COPD}=$ chronic obstructive pulmonary disease, CRGNMV = Collaborating Research Group for Noninvasive Mechanical Ventilation, RR = risk ratio. 
of weaning failures and ventilator-associated pneumonia, the length of stay in the ICU or hospital, the total duration of mechanical ventilation and the duration of invasive ventilation. Although noninvasive weaning had no effect on the duration of mechanical ventilation related to weaning, it significantly reduced tracheostomy and reintubation rates. Excluding a single quasirandomized trial supported the statistically significant reductions in mortality and ventilator-associated pneumonia rates favouring noninvasive weaning. Subgroup analyses suggested that the benefits of noninvasive weaning to mortality were significantly greater in trials exclusively enrolling patients with COPD than in trials enrolling mixed populations.

Most of the studies included in our review either exclusively or predominantly involved patients with COPD..$^{25,26,30-39}$ Our updated review adds 4 new trials to the evidence base, including
2 large trials, ${ }^{25,26} 1$ of which exclusively enrolled patients with COPD,${ }^{26}$ and 1 which predominantly enrolled patients with COPD. ${ }^{25}$ Patients with chronic airflow limitation may be ideally suited to noninvasive ventilation given its ability to offset respiratory muscle fatigue and tachypnea, augment tidal volume and reduce intrinsic positive end-expiratory pressure. Subgroup analyses for mortality suggested noninvasive weaning conferred significantly greater benefits in patients with COPD. However, inferences from subgroup analyses may be limited by the inclusion of patients with COPD in mixed population studies and the small number of trials comparing the alternative weaning strategies in patients with other causes of respiratory failure. Whether other causes of respiratory failure are as amenable as COPD to noninvasive weaning remains to be determined.

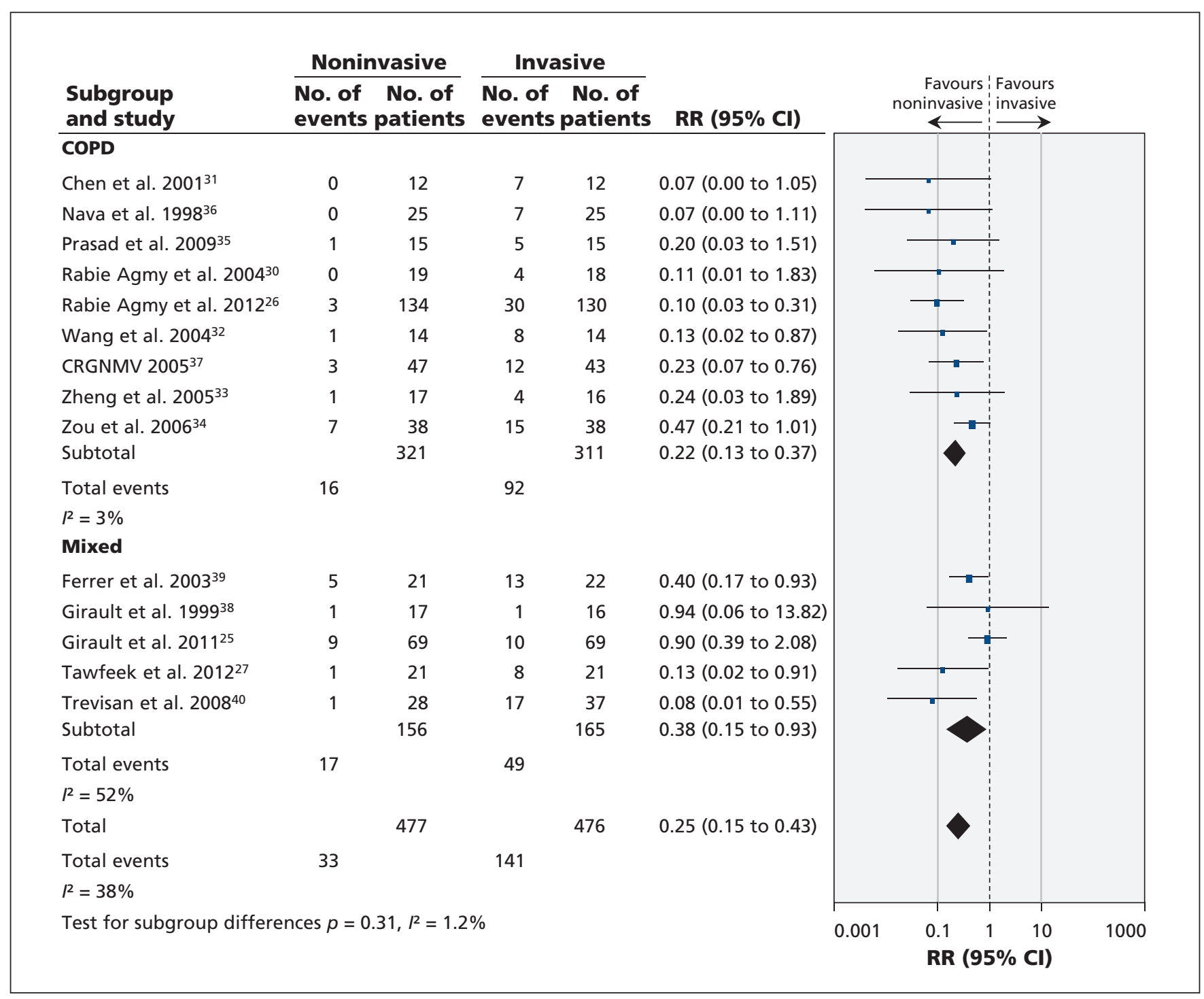

Figure 4: Effect of noninvasive weaning on ventilator associated pneumonia. $\mathrm{Cl}=$ confidence interval, COPD = chronic obstructive pulmonary disease, CRGNMV = Collaborating Research Group for Noninvasive Mechanical Ventilation, $R R=$ risk ratio. 
Overall, most of the trials in this review were of moderate quality, with 3 trials evaluated to be at low risk of bias and 2 trials considered to be at high risk of bias. The methods used to identify weaning candidates varied among trials, but occurred before randomization and are unlikely to have biased the reported duration of ventilation. Conversely, unequal or inconsistent use of weaning protocols and the frequency with which periods of spontaneous breathing (noninvasive strategy) or spontaneous breathing trials (invasive strategy) were permitted after randomization varied among the included trials. Nonstandardization of weaning protocols in unblinded trials may bias estimates of the duration of ventilation. The administration of sedation may affect the duration of ventilation, ${ }^{41}$ and only 1 trial $^{29}$ in our review used a sedation protocol.

Compared with our previous systematic review, ${ }^{16}$ our updated review contains 4 new trials ( 2 of which are large), nearly doubles the number of included patients (994 v. 530), especially those with COPD, has narrower confidence intervals around point estimates of effect and shows that noninvasive weaning reduces weaning failure and reintubation rates overall, as well as mortality in the subgroup of patients with COPD. A recent systematic review included 16 trials evaluating bilevel noninvasive ventilation and continuous positive airway pressure to wean patients on invasive ventilation, prevent respiratory failure in postoperative patients ready for extubation, or treat postextubation respiratory failure. ${ }^{15}$ Considering the population, the conclusions of that review were similar to those of ours, which included 16 trials focused on noninvasive ventilation (excluding continuous positive airway pressure) to wean patients on invasive ventilation.

\section{Strengths and limitations}

Our review was strengthened by an extensive search for relevant trials. We screened citations and abstracted data independently and in duplicate, and attempted to contact lead investigators to clarify study methods and outcomes reporting. Pooling results in a meta-analysis presupposes that the studies are sufficiently similar with respect to populations, interventions, outcome definitions and quality that one could expect a comparable underlying treatment effect. Anticipating heterogeneity across studies in pooling selected outcomes, we planned sensitivity and subgroup analyses. Furthermore, we used random-effects models, which generally give more conservative (wider) confidence intervals and consider both between-study and withinstudy variation. Finally, we reported our findings in accordance with the PRISMA (Preferred
Reporting Items for Systematic Reviews and Meta-Analyses) statement. ${ }^{42}$ In summary estimates, we found that noninvasive weaning significantly reduced mortality, length of stay in the ICU and hospital and the total duration of mechanical ventilation. These trends are consistent with, and possibly due to, reduced rates of ventilator-associated pneumonia. However, direct access to respiratory secretions among invasively weaned patients may have resulted in enhanced detection of pneumonia. The disparate mortality (range $11.1 \%$ to $60.0 \%)^{29,30,35}$ and ventilator-associated pneumonia rates $(6.3 \%$ to $59.1 \%)$ in the control group, ${ }^{38,39}$ the potential for detection bias in assessing ventilator-associated pneumonia, and the total numbers of deaths (173) and cases of ventilator-associated pneumonia (174), which are both below several hundred, ${ }^{43,44}$ may cause our effect estimates to be inflated and thereby limit the strengths of the inferences that can be drawn. Although estimates of the impact of heterogeneity associated with mortality, ventilator associated pneumonia and reintubation were moderate, those associated with most continuous outcomes were considerable; the estimates of impact of heterogeneity were unimportant for arrhythmia and tracheostomy rates. Recognizing that COPD may explain some of the heterogeneity we saw (Table 2 and Figure 2), we conducted additional post hoc secondary analyses for all study outcomes, comparing trials enrolling patients with COPD with those enrolling mixed patient populations (Appendix 5, available at www.cmaj.ca /lookup/suppl/doi:10.1503/cmaj.130974/-/DC1). Finally, in attempts to optimize the time to successful removal of invasive ventilation, clinicians are challenged by a trade-off between the risks associated with failed extubation and the complications associated with prolonged invasive ventilation. ${ }^{45}$ Clinicians may be reluctant to use noninvasive weaning owing to the need to surrender a protected airway, inexperience, concerns regarding the partial support provided by noninvasive ventilation, and the increased risk for ventilatorassociated pneumonia if reintubation is required..$^{45}$

\section{Conclusion}

Summary estimates from 16 trials suggest that noninvasive weaning reduces mortality and pneumonia without increasing the risk of weaning failure or reintubation. Moreover, in a subgroup analysis, noninvasive weaning significantly reduced mortality in studies involving patients with COPD compared with studies involving mixed populations. Our results provide the rationale to conduct a large RCT, stratified by COPD status, comparing the alternative weaning strategies. In the meantime, clinicians and cen- 
tres experienced in using noninvasive ventilation, who are currently using or considering using noninvasive ventilation for weaning patients with COPD may be reassured by our results.

\section{References}

1. Pingleton SK. Complications of acute respiratory failure. Am Rev Respir Dis 1988:137:1463-93.

2. Niederman MS, Ferranti RD, Ziegler A, et al. Respiratory infection complicating long-term tracheostomy: the implication of persistent gram-negative tracheobronchial colonization. Chest 1984;85:39-44

3. Heyland DK, Cook DJ, Griffith L, et al. The attributable morbidity and mortality of ventilator associated pneumonia in the critically ill patient. The Canadian Critical Care Trials Group. Am J Respir Crit Care Med 1999;159:1249-56.

4. MacIntyre NR, Cook DJ, Ely EW, et al. Evidence-based guidelines for weaning and discontinuing ventilatory support. A collective task force facilitated by the American College of Chest Physicians; the American Association for Respiratory Care; and the American College of Critical Care Medicine. Chest 2001; 120(Suppl 6):375S-95S

5. Appendini L, Patessio A, Zanaboni S, et al. Physiological effects of positive end expiratory pressure and mask pressure support during exacerbations of chronic obstructive pulmonary disease. Am J Respir Crit Care Med 1994;149:1069-76.

6. Nava S, Ambrosino N, Rubini F, et al. Effect of nasal pressure support ventilation and external positive end expiratory pressure on diaphragmatic function in patients with severe stable COPD Chest 1993;103:143-50.

7. Keenan SP, Sinuff T, Cook DJ, et al. Which patients with acute exacerbations of COPD benefit from noninvasive positive-pressure ventilation? A systematic review. Ann Intern Med 2003;138:861-70.

8. Peter JV, Moran JL, Phillips-Hughes J, et al. Noninvasive ventilation in acute respiratory failure: a meta-analysis update. Crit Care Med 2002;30:555-62.

9. Antonelli M, Conti G, Rocco M, et al. A comparison of noninvasive positive-pressure ventilation and conventional mechanical ventilation in patients with acute respiratory failure. $N$ Engl $\mathrm{J} \mathrm{Med}$ 1998;339:429-35.

10. Nourdine K, Combes P, Carton MJ, et al. Does noninvasive ventilation reduce the ICU nosocomial infection risk? A prospective clinical survey. Intensive Care Med 1999;25:567-73.

11. Rathgeber J, Schorn B, Falk V, et al. The influence of controlled mechanical ventilation (CMV), intermittent mandatory ventilation (IMV) and biphasic intermittent positive airway pressure (BIPAP) on duration of intubation and consumption of analgesics and sedatives. A prospective analysis of in 596 patients following adult cardiac surgery. Eur J Anaesthesiol 1997;14:576-82.

12. Criner GJ, Tzouanakis A, Kreimer DT. Overview of improving tolerance of long-term mechanical ventilation. Crit Care Clin 1994;10:845-66.

13. Mehta S, Hill NS. Noninvasive ventilation. Am J Respir Crit Care Med 2001;163:540-77.

14. Udwadia ZF, Santis GK, Steven MH, et al. Nasal ventilation to facilitate weaning in patients with chronic respiratory insufficiency. Thorax 1992;47:715-8.

15. Glossop AJ, Shepherd N, Bryden DC, et al. Non-invasive ventilation for weaning, avoiding reintubation after extubation and in the postoperative period: a meta-analysis [published erratum in Br J Anaesth 2013;110:164.] Br J Anaesth 2012;109:305-14.

16. Burns KEA, Adhikari NKJ, Keenan SP, et al. Use of noninvasive ventilation to wean critically ill adults from invasive ventilation: meta-analysis and systematic review. BMJ 2009;338:b1574.

17. Keenan SP, Sinuff T, Burns KEA, et al. as the Canadian Critical Care Trials Group/Canadian Critical Care Society Noninvasive Ventilation Guidelines Group. Clinical practice guidelines for the use of noninvasive positive-pressure ventilation and noninvasive continuous positive airway pressure in the acute care setting. CMAJ 2011;183:E195-214.

18. Review Manager (RevMan) version 5.1.6 [Computer program]. The Cochrane Collaboration. The Nordic Cochrane Centre, 2011.

19. Cochran W. The combination of estimates from different experiments. Biometrics 1954;10:101-29.

20. Berlin JA, Laird NM, Sachs HS, et al. A comparison of statistical methods for combining event rates from clinical trials. Stat Med 1989;8:141-51.

21. Higgins JP, Thompson SG. Quantifying heterogeneity in a metaanalysis. Stat Med 2002;21:1539-58.

22. Higgins JPT, Green S, editors. Cochrane Handbook of Systematic Reviews of Interventions Version 5.1.0. [updated March
2011] edition. The Cochrane Collaboration; 2011. Available: www.cochrane-handbook.org.

23. Borenstein N, Hedge LV, Higgins JPT, et al. Introduction to meta-analysis. Chichester (UK): John Wiley \& Sons; 2008.

24. Guyatt GH, Oxman AD, Vist GE, et al.; GRADE Working Group. Grade an emerging consensus on rating quality of evidence and strength of recommendations. BMJ 2008;336:924-6.

25. Girault C, Bubenheim M, Abroug F, et al. Noninvasive ventilation and weaning in patients with chronic hypercapnic respiratory failure. Am J Respir Crit Care Med 2011;184:672-9.

26. Rabie Agmy GM, Metwally MM. Noninvasive ventilation in the weaning of patients with acute-on-chronic respiratory failure due to COPD. Egyptian J Chest Dis Tuberculosis 2012;61:84-91.

27. Tawfeek MM, Ali-Elnabtity AM. Noninvasive proportional assist ventilation may be useful in weaning patients who failed a spontaneous breathing trial. Egyptian J Anaesthes 2012;28:89-94.

28. Vaschetto R, Turucz E, Dellapiazza F, et al. Noninvasive ventilation after early extubation in patients recovering from hypoxemic acute respiratory failure: a single-centre feasibility study. Intensive Care Med 2012;38:1599-606.

29. Hill NS, Lin D, Levy M, et al. Noninvasive positive pressure ventilation (NPPV) to facilitate extubation after acute respiratory failure: a feasibility study. Am J Respir Crit Care Med 2000; 161:B18.

30. Rabie Agmy GM, Mohamed AZ, Mohamed RN. Noninvasive ventilation in the weaning of patients with acute-on-chronic respiratory failure due to COPD. Chest 2004;126(Suppl 4):755.

31. Chen J, Qiu D, Tao D. Time for extubation and sequential noninvasive mechanical ventilation in COPD patients with acute exacerbated respiratory failure who received invasive ventilation [article in Chinese]. Zhongua Jie He He Hu Xi Za Zhi 2001; 24:99-100.

32. Wang X, Du X, Zhang W. Observation of the results and discussion on the timing of transition from invasive mechanical ventilation to noninvasive ventilation in COPD patients with concomitant acute respiratory failure. Shandong Med J 2004;44:4-6

33. Zheng R, Liu L, Yang Y. Prospective randomized controlled clinical study of sequential non-invasive following invasive mechanical ventilation in patients with acute respiratory failure induced COPD. Chinese J Emerg Med 2005; 14:21-5.

34. Zou SH, Zhou R, Chen P, et al. Application of sequential noninvasive following invasive mechanical ventilation in COPD patients with severe respiratory failure by investigating the appearance of pulmonary-infection-control-window [article in Chinese]. Zhong Nan Da Xue Xue Bao Yi Xue Ban 2006;31:120-4.

35. Prasad SB, Chaudhry D, Khanna R. Role of noninvasive ventilation in weaning from mechanical ventilation in patients of chronic obstructive pulmonary disease: an Indian experience. Indian J Crit Care Med 2009;13:207-12.

36. Nava S, Ambrosino N, Clini E, et al. Noninvasive mechanical ventilation in the weaning of patients with respiratory failure due to chronic obstructive pulmonary disease: a randomized, controlled trial. Ann Intern Med 1998:128:721-8.

37. Collaborating Research Group for Noninvasive Mechanical Ventilation of the Chinese Respiratory Society. Pulmonary infection control window in the treatment of severe respiratory failure of chronic obstructive pulmonary diseases: a prospective, randomized controlled, multi-centre study. Chin Med J (Engl) 2005;118: 1589-94.

38. Girault C, Daudenthun I, Chevron V, et al. Noninvasive ventilation as a systematic extubation and weaning technique in acuteon-chronic respiratory failure: a prospective, randomized controlled study. Am J Respir Crit Care Med 1999;160:86-92.

39. Ferrer M, Esquinas A, Arancibia F, et al. Noninvasive ventilation during persistent weaning failure. Am J Respir Crit Care Med 2003; 168:70-6.

40. Trevisan CE, Viera SR; the Research Group in Mechanical Ventilation Weaning. Noninvasive mechanical ventilation may be useful in treating patients who fail weaning from invasive mechanical ventilation: a randomized clinical trial. Crit Care 2008;12:R51

41. Brook AD, Ahrens TS, Schaiff R, et al. Effect of a nursingimplemented sedation protocol on the duration of mechanical ventilation. Crit Care Med 1999;27:2609-15.

42. Moher D, Liberati A, Tetzlaff J, et al.; Prisma Group. Preferred reporting items for systematic reviews and meta-analyses: the PRISMA statement. J Clin Epidemiol 2009;62:1006-12.

43. Devereaux PJ, Yusuf S, Yang H, et al. Are the recommendations to use perioperative beta-blocker therapy in patients undergoing noncardiac surgery based on reliable evidence? CMAJ 2004;171:245-7.

44. Thorlund K, Imberger G, Walsh M, et al. The number of patients and events required to limit the risk of overestimation of intervention effects in meta-analysis: a simulation study. PLOS ONE 2011;6:e25491. 
45. Pawar M, Mehta Y, Khurana P, et al. Ventilator associated pneumonia: incidence, risk factors, outcome and microbiology. $J$ Cardiothorac Vasc Anesth 2003;17:22-8.

Affiliations: Interdepartmental Division of Critical Care and the University of Toronto and the Li Ka Shing Knowledge Institute (Burns, Adhikari), Toronto, Ont.; Department of Clinical Epidemiology and Biostatistics (Meade), McMaster University, Hamilton, Ont.; Faculty of Medicine and Dentistry (Premji), University of Toronto, Toronto, Ont.; Department of Critical Care Medicine and Sunnybrook Research Institute, Sunnybrook Health Sciences Centre (Adhikari), Toronto, Ont.

Contributors: Karen Burns conducted the literature searches, screened abstracts, selected studies meeting inclusion criteria, extracted data, assessed study quality, con- ducted risk of bias assessments, prepared initial and subsequent drafts of the manuscript, and integrated comments in to revised versions of the manuscript. Neill Adhikari screened abstracts, selected studies meeting inclusion criteria, extracted data, assessed study quality, double checked data entry, and conducted risk of bias assessments. Azra Premji retrieved study articles, aided with updating the texts of the manuscript, verified summary estimates in the manuscript. Maureen Meade provided methodologic guidance and adjudicated disagreements between reviewers. All authors revised and approved the final version of the manuscript. Karen Burns is the guarantor.

Acknowledgement: Karen Burns holds a Clinician Scientist (Phase 2) Award from the Canadian Institutes of Health Research. 